\title{
AORTIC ANEURYSM: A RARE CAUSE OF ORTNER'S SYNDROME
}

Sukanta Kodali¹, Biswal Pradipta Trilochan², Swetapadma Pradhan ${ }^{3}$, Jyoti Patnaik ${ }^{4}$, Manoranjan Dash ${ }^{5}$

\section{HOW TO CITE THIS ARTICLE:}

Sukanta Kodali, Biswal Pradipta Trilochan, Swetapadma Pradhan, Jyoti Patnaik, Manoranjan Dash. "Aortic Aneurysm: A Rare Cause of Ortner's Syndrome". Journal of Evolution of Medical and Dental Sciences 2014;

Vol. 3, Issue 58, November 3; Page: 13199-13202, DOI: 10.14260/jemds/2014/3756

ABSTRACT: A 60 year old male patient presented with hoarseness of voice lasting for 2 months. First he was examined by the otorhinolaryngologist of our hospital, who noticed absence of movements of the left vocal cord. Chest X-ray showed a large opacity in the left upper zone and the patient was referred to the pulmonologist. His general physical examination was unremarkable not having any stigmata of Marfan's or Ehler Danlos syndrome. On chest auscultation, a systolic flow murmur was audible and there was decreased breath sound in the left infraclavicular area. Bronchoscopy revealed absence of movement of left vocal cord. His trans-thoracic echocardiography revealed degenerative aortic and mitral valve disease with mild aortic regurgitation and mild mitral regurgitation with dilated aortic aneurysm. A contrast enhanced CT scan of thorax and CT Angio Aortogram revealed fusiform aneurysmal dilatation of the arch of aorta with diameter of $6.2 \mathrm{~cm}$ over a length of $12.3 \mathrm{~cm}$ showing intraluminal partial thrombus with wall calcification. The patient was advised to undergo surgery. However, after detailed discussion with him and his family, they decided for medical management in view of his advanced age.

INTRODUCTION: Ortner's syndrome is a clinical entity defined as hoarseness caused by impairment of the left recurrent laryngeal nerve because of cardiovascular diseases. It was first described in 1897 by Nobert Ortner in a patient with left atrial enlargement secondary to mitral valve stenosis. Subsequently, the entity has been described in other cardiovascular diseases including mitral valve prolapse, atrial and ventricular septal defect, patent ductus arteriosus, pulmonary hypertension, and thoracic aortic aneurysm..$^{1,2}$

This syndrome is also known as cardio-vocal syndrome. ${ }^{3}$ Thoracic aortic aneurysm presenting as vocal cord paralysis is a rare occurrence. Five percent of recurrent laryngeal nerve palsy is due to thoracic aortic aneurysm. ${ }^{4}$ We present a rare case of cardio-vocal hoarseness or Ortner's syndrome secondary to aortic arch aneurysm.

CASE REPORT: A 60 year old male patient presented with hoarseness of voice lasting for 2 months. First he was examined by the otorhinolaryngologist of our hospital, who noticed absence of movements of the left vocal cord. Chest X-ray showed a large opacity in the left upper zone (Image-1) and the patient was referred to the pulmonologist. His general physical examination was unremarkable not having any stigmata of Marfan's or Ehler Danlos syndrome.

On chest auscultation, a systolic flow murmur was audible and there was decreased breath sound in the left infraclavicular area. Bronchoscopy revealed absence of movement of the left vocal cord. (Image-2) His trans-thoracic echocardiography revealed degenerative aortic and mitral valve disease with mild aortic regurgitation and mild mitral regurgitation with dilated aortic aneurysm.

A contrast enhanced CT scan of thorax and CT Angio Aortogram revealed fusiform aneurysmal dilatation of the arch of aorta with diameter of $6.2 \mathrm{~cm}$ over a length of $12.3 \mathrm{~cm}$ showing 
intraluminal partial thrombus with wall calcification (Image-3). Proximal to aneurysm, ascending thoracic aorta diameter was $3.0 \mathrm{~cm}$ and distal to aneurysm, descending thoracic aorta diameter was $3.3 \mathrm{~cm}$.

Asymmetrical sac is protruding into subaortic space possibly compressing left recurrent laryngeal nerve. Lung parenchyma appears normal except minimal infiltration near aneurysmal site. Preoperative laboratory investigations were normal and after preanaesthetic evaluation, repair of the aneurysm was planned. Our patient was advised to undergo surgery. However, after detailed discussion with him and his family, they decided for medical management in view of his advanced age.

DISCUSSION: The recurrent laryngeal nerves provide ipsilateral motor innervation to the intrinsic laryngeal muscles for vocalisation. The recurrent laryngeal nerves branch from the vagus nerve (the tenth cranial nerve) at different levels for the left and right side. The recurrent laryngeal nerves descend first into the thorax before rising up between the trachea and esophagus to reach the neck. The right recurrent laryngeal nerve branches at the level of the right subclavian artery and hooks around this artery.

The left recurrent laryngeal nerve is longer and it branches from the vagus nerve at the level of the transverse aortic arch. It hooks under the arch of aorta, posterior to the ligamentum arteriosum before ascending towards the neck between the trachea and the esophagus. Because of the anatomical course of the recurrent laryngeal nerves, the left side is more vulnerable to damage. In unilateral vocal cord palsy due to thoracic diseases, left-sided vocal cord paralysis was 1.75 times more frequent than right-sided paralysis. ${ }^{5}$

Damage to the left recurrent laryngeal nerve can happen at any level of the nerves course and in some cases it can be compressed between the aorta and pulmonary artery because of a very narrow space between these vessels. The incidence of thoracic aortic aneurysms in males has been reported to be 16.3 per 100,000 person/year and in females it is up to 9.1 per 100,000 person/ year. ${ }^{6}$ In thoracic aortic aneurysms, most commonly involved site of the thoracic aorta is the ascending thoracic aorta with or without aortic root involvement followed by the aneurysms of the descending thoracic aorta. In only $10 \%$ of the cases of thoracic aortic aneurysms, the aortic arch is involved. ${ }^{7}$

Patients having thoracic aortic aneurysms are usually asymptomatic.7,8 Thoracic aortic aneurysm presenting as vocal cord paralysis is a rare occurrence. Only five percent of recurrent laryngeal nerve palsy is due to thoracic aortic aneurysm. ${ }^{4}$ Computed tomography (CT) angiography is an ideal test for detecting thoracic aortic aneurysm, for delineating its anatomy, determining its size, defining the aortic branches, the extent of involvement and the effect on adjacent structures. ${ }^{7}$

Aortic aneurysm is a life-threatening condition if it ruptures. Some authors have advocated that the presence of recurrent laryngeal nerve palsy is a prodrome of aneurysm rupture. ${ }^{9}$ Asymptomatic aneurysms can be managed medically. Medical management includes aggressive blood pressure control and serial imaging of the aneurysm to evaluate growth and structure. Asymptomatic aneurysms that are rapidly expanding warrant a surgical management. Surgery is also indicated for those who are symptomatic or for any aneurysms that are over 50 to $60 \mathrm{~mm}$ in diameter.

Surgery for thoracic aortic aneurysm can be in the form of an open surgery or in the form of endoluminal stent aneurysm repair. The reversal of Ortner's syndrome after aneurysm repair has been described. Stoob demonstrated for the first time the reversal of Ortner's syndrome after 
endoluminal aneurysm repair. ${ }^{10}$ After the release of the recurrent laryngeal nerve compression, it is possible to regain the mobility of the vocal cord. If the vocal cord paralysis persists, or if the patient has symptoms of aspiration, then medialisation of the vocal cord can be undertaken.

\section{REFERENCES:}

1. Thirwall AS. Ortner's syndrome: a centenary review of unilateral recurrent laryngeal nerve palsy secondary to cardiothoracic disease. J Laryngol Otol. 1997; 111: 869-871.

2. Hebl JR, Rose SH, Narr BJ, Rorie DK. Postoperative left vocal cord dysfunction caused by Ortner's cardiovocal syndrome. Anesth Analg. 2001; 92: 1071-1072.

3. Plastiras SC, Pamboucas C, Zafiriou T, Lazaris N, Toumanidis S. Ortner's syndrome: a multifactorial cardiovocal syndrome. Clin Cardiol 2010; 33: 99-100.

4. Teixido MT, Leonetti JP. Recurrent laryngeal nerve paralysis associated with thoracic aortic aneurysm. Otolaryngol Head Neck Surg 1990; 102: 140-4.

5. Sun Wha Song, Beom Cho Jun, Kwang Jae Cho, Sungwon Lee, Young Joo Kim, and Seog Hee Park: "CT Evaluation of Vocal Cord Paralysis due to Thoracic Diseases: A 10-Year Retrospective Study”; Yonsei Med J. Sep 1, 2011; 52 (5): 831-837.

6. Loughran S, Alves C, MacGregor FB. Current aetiology of unilateral vocal fold paralysis in a teaching hospital in the West of Scotland. J Laryngol Otol 2002; 116: 907-10.

7. Hiratzka LF, Bakris GL, Beckman JA, Bersin RM, Carr VF, Casey DE, et al. Guidelines for the diagnosis and management of patients with thoracic aortic disease: a report of the American College of Cardiology Foundation/American Heart Association Task Force on Practice Guidelines, American Association for Thoracic Surgery, American College of Radiology, American Stroke Association, Society of Cardiovascular Anesthesiologists, Society for Cardiovascular Angiography and Interventions, Society of Interventional Radiology, Society of Thoracic Surgeons, and Society for Vascular Medicine. Circulation 2010; 121: e266-e369.

8. Pressler V, McNamara JJ. Aneurysms of the thoracic aorta: review of 260 cases. J Thorac Cardiovasc Surg 1985; 89: 50.

9. Ohki M. Thoracic saccular aortic aneurysm presenting with recurrent laryngeal nerve palsy prior to aneurysm rupture: A prodrome of thoracic aneurysm rupture. Case reports in Otolaryngology, vol. 2012, Article ID 367873, 3 pages, 2012. Doi: 10.1155/2012/367873.

10. Stoob K, Alkadhi H, Lachat M, Wildermuth S, Pfammatter T. Resolution of hoarseness after endovascular repair of thoracic aortic aneurysm: a case of Ortner's syndrome. Ann Otol Rhinol Laryngol 2004; 113 (1): 43-5.

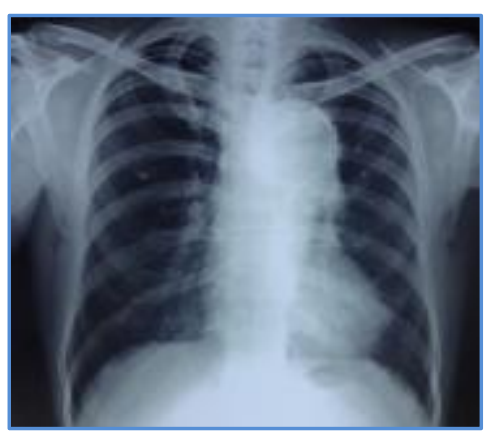

Image-1

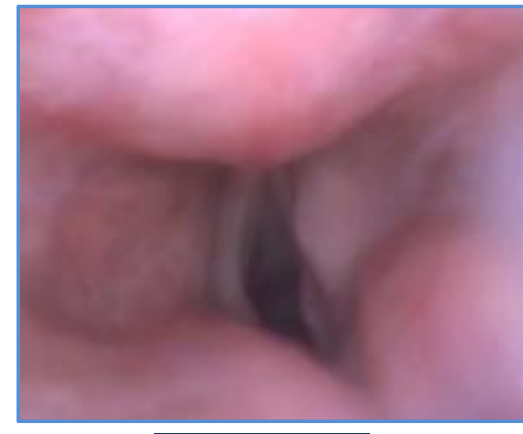

Image-2 


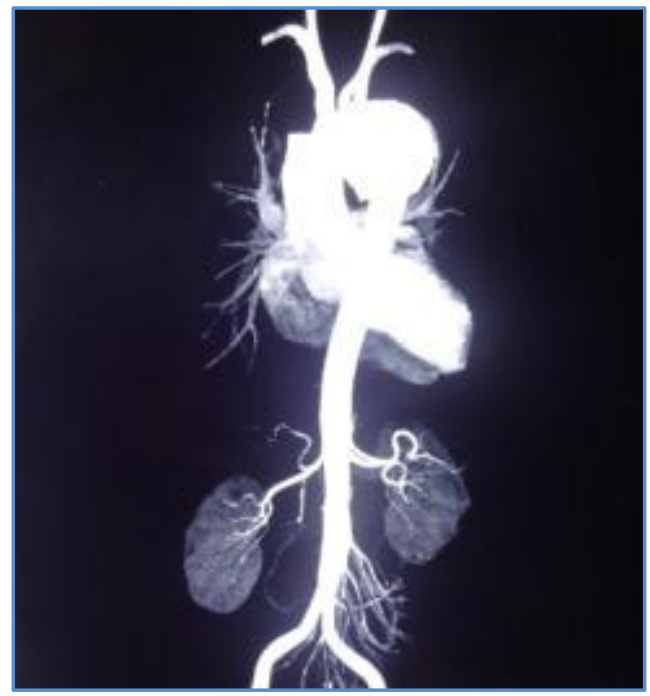

Image-3

\section{AUTHORS:}

1. Sukanta Kodali

2. Biswal Pradipta Trilochan

3. Swetapadma Pradhan

4. Jyoti Patnaik

5. Manoranjan Dash

\section{PARTICULARS OF CONTRIBUTORS:}

1. Post Graduate Student, Department of Pulmonary Medicine, S.C.B. Medical College, Cuttack.

2. Senior Resident, Department of Pulmonary Medicine, S.C.B. Medical College, Cuttack.

3. Senior Resident, Department of Pulmonary Medicine, S.C.B. Medical College, Cuttack.
4. Professor, Department of Pulmonary Medicine, S.C.B. Medical College, Cuttack.

5. Assistant Professor, Department of Pulmonary Medicine, S.C.B. Medical College, Cuttack.

\section{NAME ADDRESS EMAIL ID OF THE CORRESPONDING AUTHOR:}

Dr. Sukanta Kodali, Andul Chowdhury Para, P.O. Andul-Mouri, Howrah District, West Bengal-711302.

Email: sukantakodali@gmail.com

Date of Submission: 18/10/2014.

Date of Peer Review: 20/10/2014.

Date of Acceptance: 28/10/2014.

Date of Publishing: 03/11/2014. 